JEEP 2011, 00003 (2011)

DOI: $10.1051 /$ jeep/201100003

(C) Owned by the authors, published by EDP Sciences, 2011

\title{
Growth of single-crystal fibers for non-linear optical applications using the micro-pulling down technique ( $\mu$-PD)
}

\author{
N. Kozhaya, M. Ferriol, M. Cochez, M. Aillerie \\ Laboratoire Matériaux Optiques, Photonique et Système, E.A. 4423, Université Paul Verlaine-Metz et Supélec, \\ Département Chimie, I.U.T de Moselle-Est, 12 rue V. Demange, B.P. 80105, 57503-Saint-Avold Cédex,France
}

\begin{abstract}
The versatility of the micro-pulling down technique has been investigated through the growth of single crystal fibers of three kinds of materials presenting different behaviours on melting. The materials studied were either congruently melting $\left(\mathrm{LiNbO}_{3}\right)$, or incongruently melting through a peritectic reaction $\left(\mathrm{Ca}_{5}\left(\mathrm{BO}_{3}\right)_{3} \mathrm{~F}\right)$ or a solid solution $\left(\mathrm{K}_{3} \mathrm{Li}_{2-\mathrm{x}} \mathrm{Nb}_{5+\mathrm{x}} \mathrm{O}_{15+2 \mathrm{x}}\right)$. In each case, regular and transparent fibers free of macroscopic defects (bubbles, inclusions, cracks) could be obtained.
\end{abstract}

\section{Introduction}

The growth of single crystals of materials usable for nonlinear optical (NLO) applications can be performed by different techniques: Kyropoulos, Czochralski or HTTSSG (High Temperature Top Seeded Solution Growth). However, these methods are not adequate to grow crystals with non congruent melting (particularly crystals originating from a solid solution) as they lead to strong compositional inhomogeneities rendering the crystals unusable.

To overcome this problem, growth techniques allowing high growth rates (high axial temperature gradients) allow to obtain homogeneous crystals because, in this case, the melt and the crystal maintain the same composition and the composition remains constant throughout the crystal. Such a behaviour occurs when using fiber growth technique such as the micro-pulling down ( $\mu$-PD) [1].

In this paper, we illustrate the versatility of this technique through three examples of materials grown in our laboratory for applications in non-linear optics.

\section{Materials and preparation procedure}

We have investigated the growth conditions of three compounds: Fe-doped $\mathrm{LiNbO}_{3}$ (LN, congruent melting), $\mathrm{K}_{3} \mathrm{Li}_{2-\mathrm{x}} \mathrm{Nb}_{5+\mathrm{x}} \mathrm{O}_{15+2 \mathrm{x}}\left(\mathrm{KLN}\right.$, solid solution) and $\mathrm{Ca}_{5}\left(\mathrm{BO}_{3}\right)_{3} \mathrm{~F}$ (CBF, incongruent melting).

Preliminary to the crystal growth, powders of each compound were prepared by solid state reaction using $4 \mathrm{~N}$ purity reagents:

- for $\mathrm{LN}: \mathrm{Li}_{2} \mathrm{CO}_{3}, \mathrm{Nb}_{2} \mathrm{O}_{5}$ and $\mathrm{Fe}_{2} \mathrm{O}_{3}$

- for $\mathrm{KLN}: \mathrm{Li}_{2} \mathrm{CO}_{3}, \mathrm{~K}_{2} \mathrm{CO}_{3}$ and $\mathrm{Nb}_{2} \mathrm{O}_{5}$
- for $\mathrm{CBF}$ : $\mathrm{CaCO}_{3}, \mathrm{H}_{3} \mathrm{BO}_{3}$ and $\mathrm{CaF}_{2}$

After weighing in the appropriate or desired ratios, the reagents were thoroughly mixed in an agate mortar. The resulting mixture was pressed into pellets $25 \mathrm{~mm}$ in diameter and 2-3 $\mathrm{mm}$ in thickness. The pellets were then sintered in air between 10 and 30 hours at temperatures of 950 or $1000{ }^{\circ} \mathrm{C}$ according to the material, and, after regrinding, sintered again in the same conditions. Completeness of the ceramic synthesis was checked by $\mathrm{X}$-ray powder diffraction analysis performed at room temperature with a Bruker AXS-D8 diffractometer using $\mathrm{Cu}-\mathrm{K} \alpha$ radiation.

\section{Crystal growth and results}

All single-crystal fibers were grown in air atmosphere using the $\mu$-PD equipment installed in our laboratory [2, 3]. Crucibles with dimensions: $10 \times 5 \times 3 \mathrm{~mm}$ were made in a platinum plate (thickness: $0.07 \mathrm{~mm}$ ). The nozzle assembled to the crucible was made from a platinum tube (length: $1-2 \mathrm{~mm}$, thickness: $0.1 \mathrm{~mm}$, internal diameter: from 0.55 to $1 \mathrm{~mm}$ ).

\subsection{Fe-doped lithium niobate (LN)}

The aim of the growth of Fe-doped LN crystal fibers was to study the possibility of engraving optical waveguides for telecom applications by taking advantage of the naturally present photorefractive effect in $\operatorname{LN}[3,4]$.

Several crystal fibers up to $120 \mathrm{~mm}$ long were currently grown using an a-axis oriented seed with pulling rates in the range $0.3-2.4 \mathrm{~mm} . \mathrm{min}^{-1}$. Fig. 1 gives typical examples of the LN crystals obtained. 


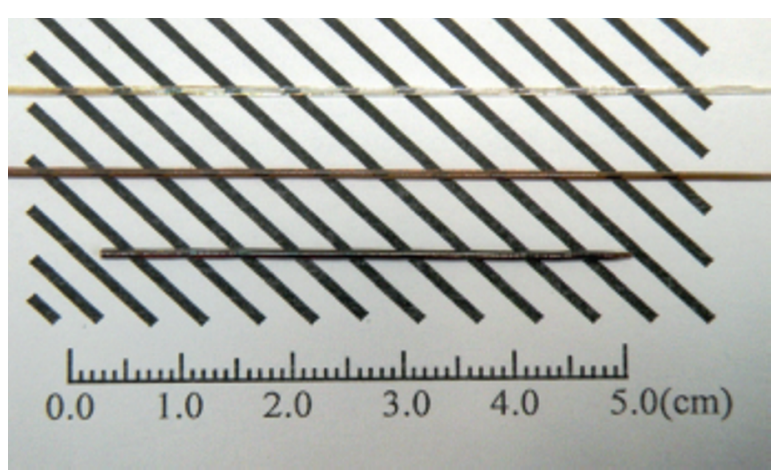

Fig. 1. $\mu$-PD grown undoped (top), Fe-doped 0.35 at $\%$ (middle) and 0.5 at $\%$ (bottom) LN single-crystal fibers

Under the optical microscope, all fibers appeared free of macro defects such as bubbles, inclusions or cracks. The axial compositional homogeneity was checked by Raman spectroscopy using a LABRAM Jobin-Yvon micro-Raman spectrometer operating with an excitation wavelength of $633 \mathrm{~nm}$. For example, Fig. 2 illustrates the spectra obtained for an a-axis oriented 0.35 at $\% \mathrm{Fe}$-doped LN fiber. The spectra were obtained with the dominant $\mathrm{Y}(\mathrm{ZZ}) \mathrm{Y}$ configuration. No evolution in frequency of all the bands can be observed all along the growth axis showing the good compositional uniformity of the crystal.

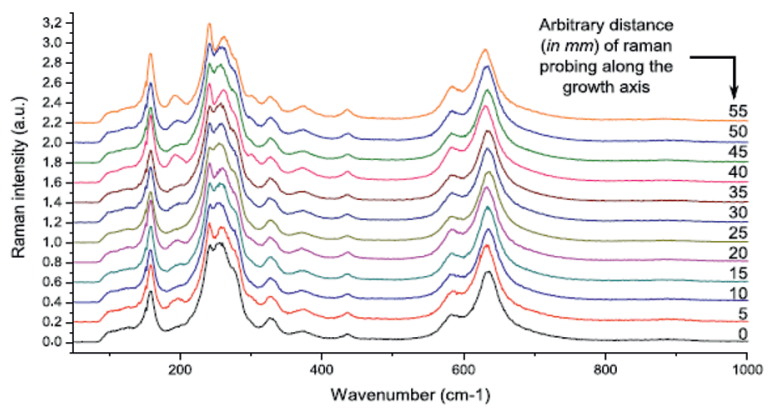

Fig. 2. Raman spectra along the growth axis of a Fe-doped $(0.35 \mathrm{at} \%)$ LN fiber showing a good compositional homogeneity

\subsection{Potassium lithium niobate (KLN)}

$\mathrm{KLN}$ is a solid solution of the ternary system $\mathrm{Li}_{2} \mathrm{O}-\mathrm{K}_{2} \mathrm{O}-$ $\mathrm{Nb}_{2} \mathrm{O}_{5}$. The only way to obtain compositionally homogeneous crystals is to grow fibers, taking advantage of the features of the $\mu$-PD method.

KLN has a number of highly desirable optical properties:

- Very high non-linear optical coefficients leading to very high conversion efficiency

- High laser damage threshold

- No hygroscopicity of crystals

- Crystals with the formula $\mathrm{K}_{3} \mathrm{Li}_{2-\mathrm{x}} \mathrm{Nb}_{5+\mathrm{x}} \mathrm{O}_{15+2 \mathrm{x}}$ can be compositionally adjusted towards the range $0 \leq \mathrm{x} \leq 0.3$ allowing noncritical phase matching at wavelengths between 760 and $790 \mathrm{~nm}$ (second harmonic generation from 380 and $485 \mathrm{~nm}$, in the blue-near UV region)
Crystal fibers were pulled using a SBN (sodium barium niobate) or KLN a-axis oriented seed, with a pulling rate equal to $0.3 \mathrm{~mm} \cdot \mathrm{min}^{-1}$. The length of the fibers was between 10 and $120 \mathrm{~mm}$ for an apparent diameter in the range $0.4-0.8 \mathrm{~mm}$. Fig. 3 gives an example of the crystals obtained.

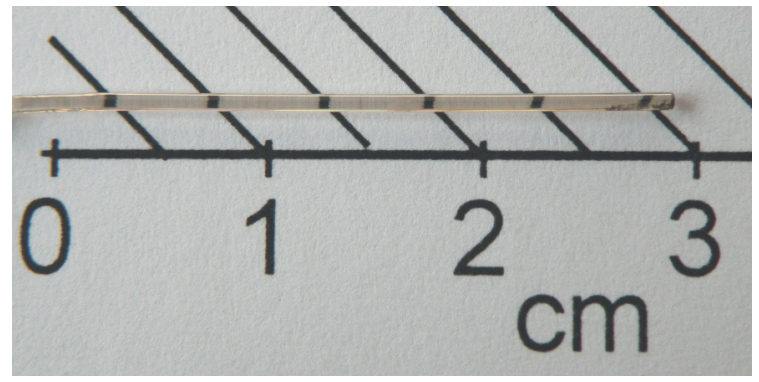

Fig. 3. Typical KLN fiber grown by the $\mu-P D$ technique

As observed for LN crystals, KLN fibers exhibited no macroscopic defects. The compositional homogeneity was also investigated by Raman micro-spectroscopy with an ARAMIS LABRAM spectrometer and with an excitation wavelength of $785 \mathrm{~nm}$. Fig. 4 gives a typical example of the spectra obtained all along the growth axis of a KLN fiber in the $\mathrm{Y}(\mathrm{ZZ}) \mathrm{Y}$ configuration. As no evolution in intensity and frequency of all the bands can be noticed, the crystal can be considered as homogeneous.

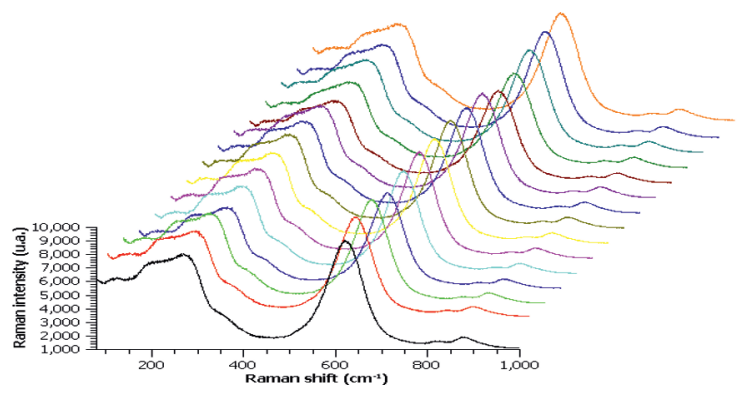

Fig. 4. Typical Raman spectra along the growth axis of a KLN crystal fiber

This is confirmed by representing the evolution of the frequency of the band near $650 \mathrm{~cm}^{-1}$ as a function of the position along the growth axis (Fig. 5). As reported earlier [5], the position of this band is very sensitive to composition variations.

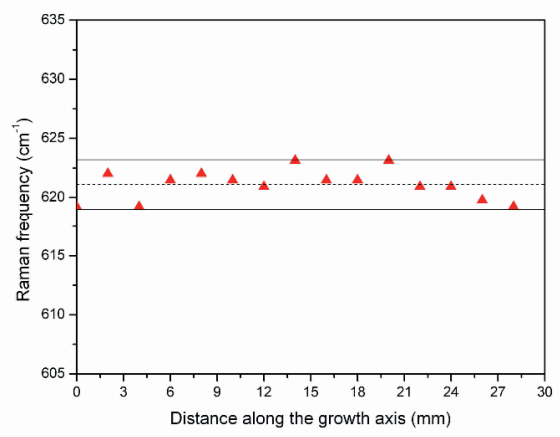

Fig. 5. Evolution of the frequency of Raman band near $650 \mathrm{~cm}^{-1}$ along the growth axis of a KLN fiber with $\mathrm{x}=0.3$ 


\subsection{Calcium fluoroborate (CBF)}

$\mathrm{CBF}$ is a promising material for UV light generation known to be not hygroscopic and chemically stable $[6,7]$. It is not congruently melting and the crystal growth can only be performed by a flux method. However, prior attempting to grow crystal fibers, we investigated more precisely the way how CBF melts. The non congruent melting of $\mathrm{CBF}$ can be the result of a peritectic reaction $(<\mathrm{CBF}>\leftrightarrows$ Liq. $+<$ solid phase $1>+<$ solid phase $2>)$ quasi-peritectic reaction $(<\mathrm{CBF}>+<$ solid phase $1>\leftrightarrows$ Liq. $+<$ solid phase $2>$ ) or incongruent melting of a stoichiometric ternary compound $(<\mathrm{CBF}>\leftrightarrows$ Liq. + $<$ solid phase $>$ ). For crystal growth purposes, it is interesting to know by which type of reaction melting occurs.

\subsubsection{Partial study of the $\mathrm{CaF}_{2}-\mathrm{CaO}-\mathrm{B}_{2} \mathrm{O}_{3}$ ternary system}

We examined the thermal behaviour of three samples selected in the $\mathrm{CaF}_{2}-\mathrm{Ca}_{3}\left(\mathrm{BO}_{3}\right)_{2}$ join of the ternary system $\mathrm{CaF}_{2}-\mathrm{CaO}-\mathrm{B}_{2} \mathrm{O}_{3}$. The composition of the samples and the results of DTA experiments are given in Table 1.

Table 1. DTA results in the $\mathrm{CaF}_{2}-\mathrm{Ca}_{3}\left(\mathrm{BO}_{3}\right)_{2}$ vertical section of the ternary system $\mathrm{CaF}_{2}-\mathrm{CaO}-\mathrm{B}_{2} \mathrm{O}_{3}$

\begin{tabular}{|c|c|c|c|c|c|c|}
\hline $\begin{array}{l}\mathrm{CaO} \\
(\mathrm{mol} \%)\end{array}$ & $\begin{array}{l}\mathrm{B}_{2} \mathrm{O}_{3} \\
(\mathrm{~mol} \%)\end{array}$ & $\begin{array}{l}\text { Peak } \\
\left({ }^{\circ} \mathrm{C}\right)\end{array}$ & 1 & $\begin{array}{l}\text { Peak } \\
\left({ }^{\circ} \mathrm{C}\right)\end{array}$ & 2 & $\begin{array}{l}\text { Peak } \\
\left({ }^{\circ} \mathrm{C}\right)\end{array}$ \\
\hline 66.00 & 22.00 & 1272 & & 1365 & & 1410 \\
\hline 66.00 & 22.00 & 1280 & & 1373 & & 1415 \\
\hline 69.23 & 23.08 & 1283 & & - & & - \\
\hline 69.23 & 23.08 & 1273 & & 1368 & & 1413 \\
\hline 72.00 & 24.00 & 1282 & & 1375 & & 1460 \\
\hline 72.00 & 24.00 & 1278 & & 1382 & & 1465 \\
\hline 72.00 & 24.00 & 1274 & & 1387 & & 1472 \\
\hline $75.00^{\mathrm{a}}$ & $25.00^{\mathrm{a}}$ & - & & - & & $1479^{a}$ \\
\hline
\end{tabular}

${ }^{\mathrm{a}}$ : from ref. [8]

In the ternary sytem, $\mathrm{CBF}$ is located near the limiting binary phase diagram $\mathrm{CaO}-\mathrm{B}_{2} \mathrm{O}_{3}$. From the previous studies or calculation of this system and from the binary diagram $\mathrm{CaF}_{2}-\mathrm{CaO}[8,9]$, it is obvious that near $\mathrm{CBF}$, only one univariant line can exist in the ternary system, originating at the eutectic point (about $76 \mathrm{CaO} \mathrm{mol} \%$, $1460{ }^{\circ} \mathrm{C}$ ) between $\mathrm{Ca}_{3}\left(\mathrm{BO}_{3}\right)_{2}$ and $\mathrm{CaO}$. Then, the only way for incongruent melting of $\mathrm{CBF}$ is through the peritectic reaction:

$\mathrm{Ca}_{5}\left(\mathrm{BO}_{3}\right)_{3} \mathrm{~F}(\mathrm{CBF}) \leftrightarrows$ Liq. $+\mathrm{CaO}+\mathrm{Ca}_{3}\left(\mathrm{BO}_{3}\right)_{2}$

The X-ray powder diffraction patterns of the three samples studied are given in Fig. 6. It shows the presence of a single phase for the sample 1 (excluding weak extra peaks corresponding to impurities originated from the preparation procedure of $\mathrm{CBF}$ ). Three phases: $\mathrm{CaO}, \mathrm{CBF}$ and $\mathrm{Ca}_{3}\left(\mathrm{BO}_{3}\right)_{2}$ were observed in the sample 2 whereas $\mathrm{CBF}$ and $\mathrm{CaF}_{2}$ were detected in the sample 3. The phase identification was performed on the basis of the ICSD patterns of CBF (PDF 01-080-1702), $\mathrm{Ca}_{3}\left(\mathrm{BO}_{3}\right)_{2}$ (PDF 01-
070-0868), $\mathrm{CaO}$ (PDF 01-070-4068) and $\mathrm{CaF}_{2}$ (PDF 03065-0535).

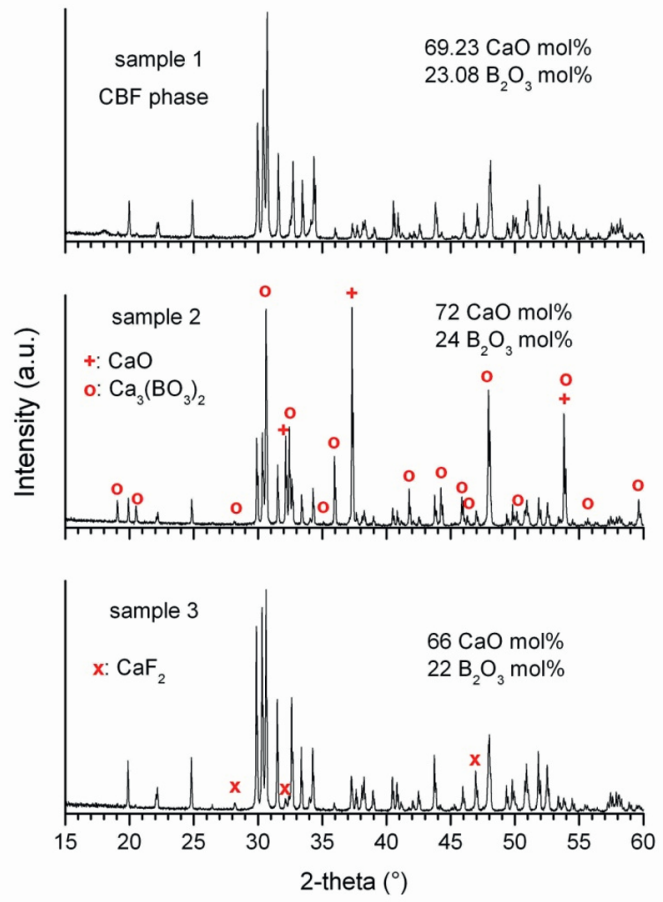

Fig. 6. Room temperature $\mathrm{X}$-ray powder diffraction patterns of the three samples belonging to the $\mathrm{CaF}_{2}-\mathrm{Ca}_{3}\left(\mathrm{BO}_{3}\right)_{2}$ join (only the extra peaks compared with those of $\mathrm{CBF}$ are indicated)

Taking these results into account, the experimental data of Table 1 lead to the representation of the vertical section given in Fig. 7.

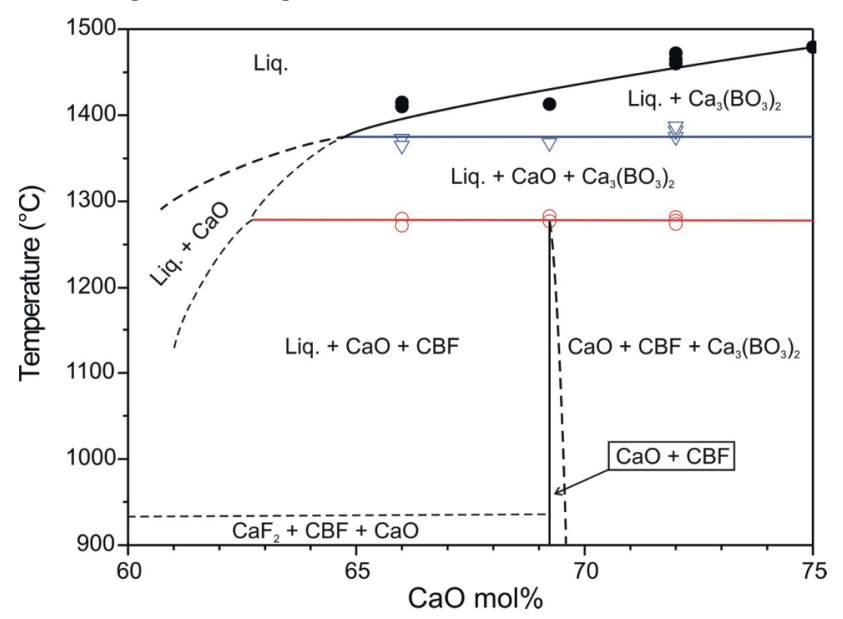

Fig. 7. Representation of the $\mathrm{CaF}_{2}-\mathrm{Ca}_{3}\left(\mathrm{BO}_{3}\right)_{2}$ vertical section in the neighbourhood of $\mathrm{CBF}$

The peritectic reaction occurs at $1277 \pm 5^{\circ} \mathrm{C}$. That representation is consistent with the room temperature powder X-ray diffraction patterns of the three samples as no phase transition was observed up to $900{ }^{\circ} \mathrm{C}$. For the sake of consistency with the construction rules of phase diagrams, the existence of a small solid solution range for CBF has to be considered, one of its limiting composition corresponding to the CBF stoichiometry. We can also see that $\mathrm{CaO}$ was not detected in the diffraction pattern of the sample 3 , its amount being very probably too low to be 
detected. This is not the case for the sample 2, for which $\mathrm{CaF}_{2}$ is still detectable. Concerning the DTA results, it should be noticed that the separation of the two last endothermal events (boundary between the three and two phase domains (both involving liquid) and the end of melting) was not easy because of their spreading nature and some overlapping combined with small thermal effects due to small amounts of the disappearing phase. However, DTA curves on cooling left no doubt on their existence as all thermal events were well separated because of strong undercooling, as shown in Fig. 8. The tilt of the second DTA peak is caused by the recalescence phenomenon due to the strong undercooling of the sample.

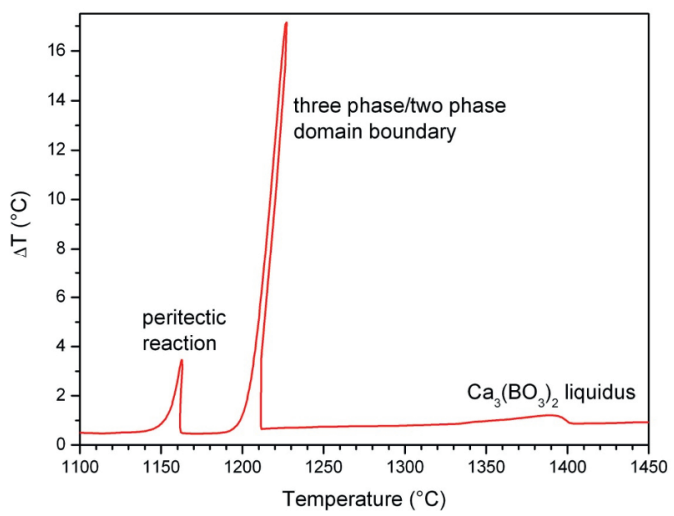

Fig. 8. DTA cooling curves for CBF phase showing the phase transitions and invariant reaction involved

\subsubsection{CBF crystal fibers growth}

CBF fibers were pulled using a c-axis oriented seed, in air atmosphereand with pulling rates between 0.05 and $0.15 \mathrm{~mm} \cdot \mathrm{min}^{-1}$. The flux had the composition: $70 \% \mathrm{CBF}$, $22.5 \% \mathrm{LiF}$ and $7.5 \% \mathrm{~B}_{2} \mathrm{O}_{3}$ to prevent excessive $\mathrm{LiF}$ evaporation [10].

Several fibers with lengths ranging from 10 to $40 \mathrm{~mm}$ were successfully grown but, the obtention of transparent fibers required the use of a nozzle with a diameter about 0.9-1 $\mathrm{mm}$ at the growth rate of $0.05 \mathrm{~mm} \cdot \mathrm{min}^{-1}$ [11] Otherwise, the crystals obtained were not transparent and irregular in diameter. It was found that this phenomenon could be related to velocity and volume flow in the nozzle too low towards the pulling rate applied. The too low flow would be due to the high viscosity of the borate melt [11]. Fig. 9 gives an example of a CBF fiber obtained.

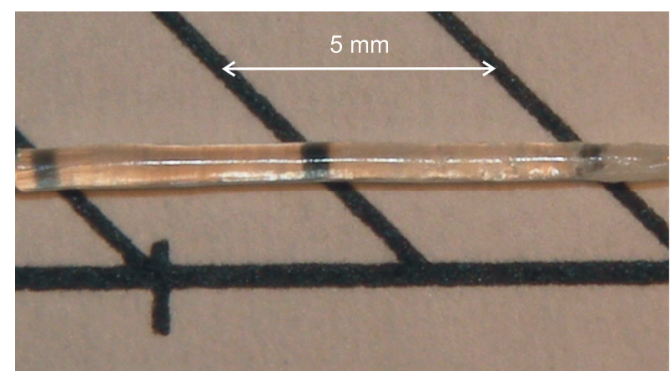

Fig. 9. Transparent CBF fiber pulled at $0.05 \mathrm{~mm} \cdot \mathrm{min}^{-1}$
Whatever the optical quality may be, all the pulled fibers were compositionally homogeneous as shown by the absence of evolution of the Raman spectra along the growth axis (Fig. 10).

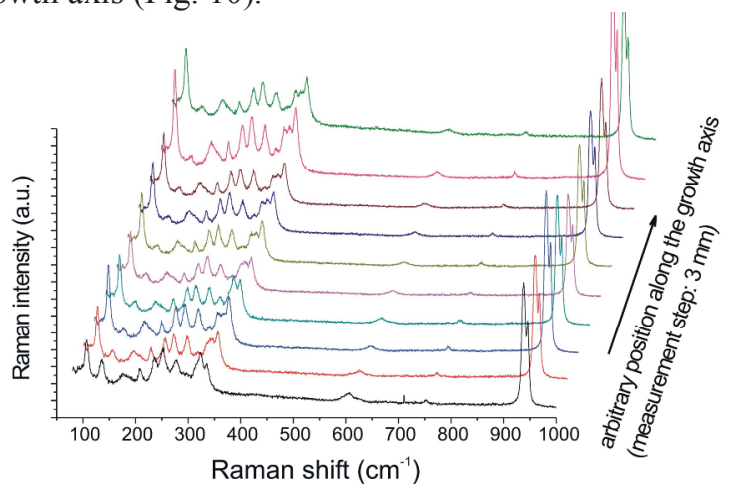

Fig. 10. Typical Raman spectra along the growth axis of a CBF crystal fiber pulled at $0.15 \mathrm{~mm} \cdot \mathrm{min}^{-1}$

\section{Conclusion}

In this work, the versatility of the micro-pulling down technique has been illustrated through three typical examples dealing with doped congruently melting compounds (LN), incongruently melting compounds requiring the use of a flux $(\mathrm{CBF})$ and solid solution compounds $(\mathrm{KLN})$. In each case, regular, transparent, macro defect free and compositionally homogeneous crystals were obtained.

\section{References}

1. D.H. Yoon, M. Hashimoto, T. Fukuda, Jpn. J. Appl. Phys., 33, 3510 (1994)

2. T. Fukuda, V.I. Chani, Shaped Crystals: Growth by Micro-Pulling Down Technique, Advances in Materials Research, vol. 8 (Springer, Berlin, 2007)

3. A. Novoselov, A. Ruso, M. Ferriol, M. Cochez, N. Fressengeas, M. Aillerie, Opt. Mat., 32, 456 (2010)

4. A. Ruso, M. Aillerie, N. Fressengeas, M. Ferriol, Appl. Phys. B, 95, 573 (2009)

5. W. Fortin, G.E. Kugel, D. Rytz, Ferroelectrics, 202, 183 (1997)

6. K. Xu, P. Loiseau, G. Aka, J. Lejay, Cryst. Growth Des., 9, 2235 (2009)

7. G. Chen, Y. Wu, P. Fu, J. Cryst. Growth, 292, 449 (2006)

8. Y. Hao, C. Qing, J. Zhanpeng, Calphad, 23, 101 (1999)

9. W.G. Seo, D. Zhou, T. Hamano, F. Tsukihashi, VII International Conference on Molten Slags, Fluxes and Salts. The South African Institute of Mining and Metallurgy, 836 (2004)

10. K. Xu, L. Gheorghe, P. Loiseau, G. Aka et al., Thematic Meeting of the CNRS/CMDO Network, http://cmdo.cnrs.fr/IMG/pdf/Loiseau_CMDO_Palais eau nov 08.pdf

11. N. Kozhaya, M. Ferriol, M. Cochez, M. Aillerie,Opt. Mater. (2011), doi:10.1016/j.optmat.2011.04.012 